\title{
The 11-element case of Frankl's conjecture
}

\author{
Ivica Bošnjak and Petar Marković * \\ Department of Mathematics and Informatics \\ University of Novi Sad, Serbia \\ ivb@im.ns.ac.yu \\ pera@im.ns.ac.yu
}

Submitted: Jan 24, 2007; Accepted: Jun 27, 2008; Published: Jul 6, 2008

Mathematics Subject Classifications: primary 05D05, secondary 05A05

\begin{abstract}
In 1979 , P. Frankl conjectured that in a finite union-closed family $\mathcal{F}$ of finite sets, $\mathcal{F} \neq\{\emptyset\}$, there has to be an element that belongs to at least half of the sets in $\mathcal{F}$. We prove this when $|\bigcup \mathcal{F}| \leq 11$.
\end{abstract}

\section{Introduction}

Frankl's conjecture [9], sometimes also called the union-closed sets conjecture is one of the most celebrated open problems in combinatorics. In [10] it is referred to as 'diabolical', presumably since it has an elementary, even trivial statement, but seems to be quite difficult. In its original statement, the conjecture is that in a finite union-closed family $\mathcal{F}$ of finite sets, $\mathcal{F} \neq\{\emptyset\}$ there has to be an element that belongs to at least half of the sets in $\mathcal{F}$. Several equivalents have been found, in various areas of mathematics, the most popular of which is probably the lattice-theoretic one (see [10], Chapter 3, Problem 39a).

Recently there have been quite a few new partial results concerning the original version of the problem, (see for instance [2], [3], [6], [7], [11, 12, 13]). Many of these papers are using the idea introduced first in [8], Theorem 1. This is a way for rapid verification of the conjecture for a large class of union-closed families using a weight function. We use a similar approach, introduced in [6]. The main difference is that Theorem 1 of [8] gives a necessary and sufficient condition for a subfamily $\mathcal{F}^{\prime}$ to force that an element of $\bigcup \mathcal{F}^{\prime}$ is in at least half of the sets of $\mathcal{F}$ for any $\mathcal{F} \supseteq \mathcal{F}^{\prime}$ (such $\mathcal{F}^{\prime}$ are called $F C$ families in [11]), while our (easier) Lemma 2.1 gives a necessary and sufficient condition for $\mathcal{F}$ to satisfy Frankl's Conjecture. We are able to use our approach to prove that any counterexample $\mathcal{F}$ to Frankl's Conjecture must satisfy $|\cup \mathcal{F}| \geq 12$.

${ }^{*}$ The second author was supported by the grant no. 144011G of the Ministry of Science and Environment of Serbia. 
In Section 2 we prove lemmas we will need later on, and which are true in any unionclosed family. Many of these are proved elsewhere, and some were left to the reader to verify in the papers where they appeared. Our goal was to have every step in our proof verifiable, so we (re-)proved the lemmas of the second kind. Section 3 consists of lemmas in the setting $|\bigcup \mathcal{F}|=11$, culminating with Theorem 3.1, which claims that all union-closed families $\mathcal{F}$ with $|\cup \mathcal{F}|=11$ satisfy Frankl's conjecture. Clearly, if there was a counterexample with $|\bigcup \mathcal{F}|<11$, we could easily construct a counterexample with $|\bigcup \mathcal{F}|=11$ by 'copying' one element into an appropriate number of 'copies' which appear in sets whenever the 'original' one does. Therefore, we prove that all union-closed families with $|\bigcup \mathcal{F}| \leq 11$ satisfy Frankl's conjecture.

\section{Initial Results}

Throughout this paper $\mathcal{F}$ will denote a finite family of finite sets closed under unions and $X$ will denote the union of $\mathcal{F}$. We will call $\mathcal{F}$ Frankl's if $X=\bigcup \mathcal{F}$ contains an element which is in at least one half of the sets from $\mathcal{F}$.

Definition 2.1. We call any function $w: X \rightarrow\{x \in R \mid x \geq 0\}$, such that $w(a)>0$ for

some $a \in X$, a weight function. The weight $w(S)$, for $S \subseteq X$ is equal to $\sum_{x \in S} w(x)$. The number $0.5 w(X)$ will be called the target weight and denoted by $t(w)$.

Lemma 2.1. $\mathcal{F}$ is Frankl's if and only if there is a weight function $w$ assigned to elements of $X=\bigcup \mathcal{F}$ such that

$$
\sum_{S \in \mathcal{F}} w(S) \geq t(w)|\mathcal{F}|
$$

Proof. $(\Longrightarrow)$ Let $a$ be an element of at least half of the sets in $\mathcal{F}$. Take the weight function $w$ such that $w(a)=1$ and $w(x)=0$ for $x \neq a$. Then $t(w)=0.5$, and the inequality is obviously satisfied.

$(\Longleftarrow)$ Assume that $\mathcal{F}$ is not Frankl's. Let $n_{a}(\mathcal{F})$ be the number of occurrences of the element $a$ in sets from $\mathcal{F}$. We take an arbitrary weight function $w$. Then

$$
\begin{gathered}
\sum_{S \in \mathcal{F}} w(S)=\sum_{S \in \mathcal{F}} \sum_{a \in S} w(a)=\sum_{a \in X} w(a) n_{a}(\mathcal{F})< \\
\sum_{a \in X} w(a) \frac{|\mathcal{F}|}{2}=t(w)|\mathcal{F}| .
\end{gathered}
$$

Lemma 2.2. If $\mathcal{F}$ contains a one-element set, or a two-element set, then it is Frankl's.

Proof. Easy exercise for the reader, and also found in several of the papers in the bibliography. 
Definition 2.2. For $S, K \subseteq X, S \cap K=\emptyset$ we call any interval in the Boolean lattice $\mathcal{P}(X)$ of the form $[K, K \cup S]$ an $S$-hypercube. We can partition a hypercube into levels, where a set is on level $k$ if and only if $k$ is the cardinality of its intersection with $S$. We denote level $k$ of a hypercube $\mathcal{C}$ by $\mathcal{C}_{k}$. Also, for $x \in S$ we define the auxiliary hypercubes $\mathcal{C}^{x}$ and $\mathcal{C}^{-x}$ to be the $S \backslash\{x\}$-hypercubes with bottom sets $K \cup\{x\}$ and $K$, respectively.

Let $\mathcal{F}$ be a union-closed family of sets and $w$ a weight function. The deficit of a set $L \subseteq X$ with $w(L)<t(w)$ is $d(L)=t(w)-w(L)$. The surplus of a set $L \subseteq X$ with $w(L)>t(w)$ is $s(L)=w(L)-t(w)$. Let $\mathcal{C}$ be an $S$-hypercube. The deficit of $\mathcal{C}$ is defined to be $d(\mathcal{C})=\sum_{\substack{L \in \mathcal{C} \mathcal{F} \\ w(L)<t(w)}} d(L)$, while $s(\mathcal{C})=\sum_{\substack{L \in \mathcal{C} \mathcal{\mathcal { F }} \\ w(L)>t(w)}} s(L)$ is the surplus of $\mathcal{C}$. Analogously we define $d\left(\mathcal{C}_{k}\right)$ and $s\left(\mathcal{C}_{k}\right)$.

It is an obvious consequence of Lemma 2.1 that if for some weight function $w$ the sum of surpluses of the sets in $\mathcal{F}$ which have weights greater than $t(w)$ is greater than or equal to the sum of deficits of the sets in $\mathcal{F}$ which have weights less than $t(w)$, then $\mathcal{F}$ is Frankl's. In particular, if for every $S$-hypercube $\mathcal{C}, s(\mathcal{C}) \geq d(\mathcal{C})$, then $\mathcal{F}$ is Frankl's. In all the $S$-hypercubes we will consider, we will have $S \in \mathcal{F}$. Hence, if the hypercube has a nonempty intersection with $\mathcal{F}$, then the top set of that hypercube is in $\mathcal{F}$.

Let $\mathcal{F}$ be a union-closed family of sets and $\mathcal{C}$ an $S$-hypercube for some $S \subseteq X$. By $p_{k}(\mathcal{C})$ we will denote the number of sets on level $k$ in the hypercube $\mathcal{C}$ which belong to $\mathcal{F}$. When $\mathcal{C}$ is obvious we will just write $p_{k}$.

Lemma 2.3. Let $\mathcal{F}$ be a union-closed family of sets and $\mathcal{C}$ an $S$-hypercube for some $S \subseteq X,|S|=m$. If $k<l<m$, suppose that for every set from level $l$ of $\mathcal{C}$ which is in $\mathcal{F}$, at most $u$ of its subsets from level $k$ could be in $\mathcal{F}$, and for every set from level $l$ of $\mathcal{C}$ which is not in $\mathcal{F}$, at most $v$ of its subsets from level $k$ could be in $\mathcal{F}$. Then

$$
\left(\begin{array}{c}
m-k \\
l-k
\end{array}\right) p_{k} \leq u p_{l}+v\left(\left(\begin{array}{c}
m \\
l
\end{array}\right)-p_{l}\right)
$$

Proof. Consider a bipartite graph $G$ whose set of vertices is $A \cup B$, where $A$ contains all $l$-level sets of $\mathcal{C}$, and $B$ contains those $k$-level sets of $\mathcal{C}$ which are in $\mathcal{F}$. Every vertex from $A$ is connected by an edge to all its subsets from $B$. Since the degree of every vertex from $B$ is $\left(\begin{array}{c}m-k \\ l-k\end{array}\right)$, this graph has $\left(\begin{array}{c}m-k \\ l-k\end{array}\right) p_{k}$ edges. On the other hand, for all sets from $A$ which are not in $\mathcal{F}$, their degree is not more than v, and the degree of those $A$-sets which are in $\mathcal{F}$ is not greater than $u$. From these facts we conclude

$$
\left(\begin{array}{c}
m-k \\
l-k
\end{array}\right) p_{k} \leq u p_{l}+v\left(\left(\begin{array}{c}
m \\
l
\end{array}\right)-p_{l}\right) .
$$

In the special case when $l=k+1$ and the number of level $k$-subsets in $\mathcal{F}$ is not limited, we have $u=k+1$ and $v=1$, so

$$
(m-k) p_{k} \leq k p_{k+1}+\left(\begin{array}{c}
m \\
k+1
\end{array}\right) .
$$

Inequality (2) is equivalent to Lemma 3.4. (b) from [11]. 
Proposition 2.1. Assume that $\mathcal{F}$ contains three different three-element sets which are all subsets of the same four-element set. Then $\mathcal{F}$ is Frankl's.

Proof. This was proved in [8], Corollary 4.

Proposition 2.2. Suppose that $\mathcal{F}$ contains three three-element sets which all contain the same two elements. Then $\mathcal{F}$ is Frankl's.

Proof. See [12], Section 3 and [6], Proposition 2.2.

The following proposition can be found in [7], with the sketch of a proof.

Proposition 2.3. [7] Let $\{a, b, c, d, e\} \subseteq X,\{a, b, c\},\{a, b, d\},\{c, d, e\} \in \mathcal{F}$. Then $\mathcal{F}$ is Frankl's.

Proof. Assume that $\mathcal{F}$ is not Frankl's. As suggested in [7], we choose the weight function $w$ such that $w(a)=w(b)=w(c)=w(d)=2, w(e)=1$, and $w(x)=0$ for all other $x \in X$. Consider an arbitrary $\{a, b, c, d, e\}$-hypercube $\mathcal{C}$ with bottom set $K$. Let us consider $\mathcal{C}_{1} \cup \mathcal{C}_{4}$. Here $K \cup\{a\} \in \mathcal{F}$ implies $K \cup\{a, c, d, e\} \in \mathcal{F}, K \cup\{b\} \in \mathcal{F}$ implies $K \cup\{b, c, d, e\} \in \mathcal{F}, K \cup\{e\} \in \mathcal{F}$ implies $K \cup\{a, b, c, e\} \in \mathcal{F}$ and $K \cup\{a, b, d, e\} \in \mathcal{F}$. This means that $d\left(\mathcal{C}_{1}\right)>s\left(\mathcal{C}_{4}\right)$ only if $K \cup\{c\}, K \cup\{d\} \in \mathcal{F}$ (and, therefore $K \cup\{a, b, c, d\} \in \mathcal{F}$ ), $K \cup\{a, b, c, e\}, K \cup\{a, b, d, e\} \notin \mathcal{F}$, and in this case $d\left(\mathcal{C}_{1}\right)=s\left(\mathcal{C}_{4}\right)+1.5$. On levels 2 and 3 we have the following situation: If $p_{1}\left(\mathcal{C}^{e}\right)=3$ then $p_{2}\left(\mathcal{C}^{e}\right) \geq 3$ and if $p_{1}\left(\mathcal{C}^{e}\right)=4$ then $p_{2}\left(\mathcal{C}^{e}\right)=6$. This means $d\left(\mathcal{C}_{1}^{e}\right) \leq s\left(\mathcal{C}_{2}^{e}\right)+3$. Also, if $K \in \mathcal{F}$, since $K \cup\{a, b, c\}$, $K \cup\{a, b, d\} \in \mathcal{F}$, and $p_{2}\left(\mathcal{C}^{\urcorner e}\right) \geq 4$ implies $\left.p_{3}(\mathcal{C}\urcorner e\right) \geq 3$, we have $d\left(\mathcal{C}_{2}^{\neg e}\right) \leq s\left(\mathcal{C}_{3}{ }^{e}\right)-1.5$. On the other hand, if $K \notin \mathcal{F}$, then $d\left(\mathcal{C}_{2}^{\neg e}\right) \leq s\left(\mathcal{C}_{3}^{\neg e}\right)+0.5$, the equality being achieved only when $K \cup\{c, d\}$ is the only set from $\mathcal{C}_{2}$ in $\mathcal{F}$, and $p_{3}\left(\mathcal{C}^{e}\right)=0$. The levels 0 and 5 of $\mathcal{C}$ produce a surplus of 4.5 when $K \notin \mathcal{F}$ and cancel each other when $K \in \mathcal{F}$.

The analysis from above guarantees that when $K \notin \mathcal{F}$, then $s(\mathcal{C})<d(\mathcal{C})$ only if $d\left(\mathcal{C}_{1}\right)=s\left(\mathcal{C}_{4}\right)+1.5$ and $d\left(\mathcal{C}_{2}^{\neg e}\right)=s\left(\mathcal{C}_{3}^{\neg e}\right)+0.5$. The first requires $K \cup\{c\} \in \mathcal{F}$, and the second requires $\left.p_{3}(\mathcal{C}\urcorner e\right)=0$. These two requirements are incompatible in any union-closed system $\mathcal{F}$ which contains $\{a, b, c\}$. So we may assume $K \in \mathcal{F}$. We will discuss three cases.

1. $K \cup\{a, b, c, e\}, K \cup\{a, b, d, e\} \notin \mathcal{F}$. This means $p_{1}\left(\mathcal{C}^{e}\right)=0$ and we have $d\left(\mathcal{C}_{1}\right) \leq$ $s\left(\mathcal{C}_{4}\right)+1.5, d\left(\mathcal{C}_{1}^{e}\right) \leq s\left(\mathcal{C}_{2}^{e}\right)-0.5$ and $d\left(\mathcal{C}_{2}^{\neg e}\right) \leq s\left(\mathcal{C}_{3}^{\neg e}\right)-1.5$. Finally, this gives $d(\mathcal{C}) \leq s(\mathcal{C})-0.5$

2. $K \cup\{a, b, c, e\} \in \mathcal{F}, K \cup\{a, b, d, e\} \notin \mathcal{F}$. This means $p_{1}\left(\mathcal{C}^{e}\right) \leq 1$ and we have $d\left(\mathcal{C}_{1}\right) \leq s\left(\mathcal{C}_{4}\right)-1, d\left(\mathcal{C}_{1}^{e}\right) \leq s\left(\mathcal{C}_{2}^{e}\right)+1$ and $d\left(\mathcal{C}_{2}^{\neg e}\right) \leq s\left(\mathcal{C}_{3}{ }^{e}\right)-1.5$. Finally, this gives $d(\mathcal{C}) \leq s(\mathcal{C})-1.5$.

3. $K \cup\{a, b, c, e\}, K \cup\{a, b, d, e\} \in \mathcal{F}$. If $s(\mathcal{C})<d(\mathcal{C})$, then $K \cup\{c\}, K \cup\{d\}, K \cup\{e\}$ must be in $\mathcal{F}$. Now we analyze the sets in $\mathcal{C}$ in a different way. $K \cup\{c\}, K \cup\{d\}$ and $K \cup\{e\}$ cancel out with $K \cup\{a, b, c, e\}, K \cup\{a, b, d, e\}$ and $K \cup\{a, b, c, d\} . K \cup\{c, e\}$ and $K \cup\{d, e\}$ cancel out with $K \cup\{a, b, c\}$ and $K \cup\{a, b, d\}$. As $K \cup\{e\} \in \mathcal{F}$, $d\left(\mathcal{C}_{2}^{\neg e}\right) \leq s\left(\mathcal{C}_{2}^{e}\right)$. Since $K \cup\{a\} \in \mathcal{F}$ implies $K \cup\{a, c, d\}, K \cup\{a, c, d, e\} \in \mathcal{F}$, and 
$K \cup\{a, e\} \in \mathcal{F}$ implies $K \cup\{a, c, d, e\} \in \mathcal{F}$, sets $K \cup\{a\}$ and $K \cup\{a, e\}$ cancel out with $K \cup\{a, c, d\}$ and $K \cup\{a, c, d, e\}$. Similarly, $K \cup\{b\}$ and $K \cup\{b, e\}$ cancel out with $K \cup\{b, c, d\}$ and $K \cup\{b, c, d, e\}$. This gives $d(\mathcal{C}) \leq s(\mathcal{C})$.

Theorem 2.1. [7] Assume that $\mathcal{F}$ contains three different three-element sets which are all subsets of the same five-element set. Then $\mathcal{F}$ is Frankl's.

Proof. There are four possible cases:

1. $\mathcal{F}$ contains three three-element subsets of a four-element set. This case is considered in Proposition 2.1.

2. $\mathcal{F}$ contains three three-element sets which all contain the same two elements. The statement holds by Proposition 2.2 .

3. $\mathcal{F}$ contains three three-element sets whose union is a five-element set and whose intersection is a one-element set. This case is solved in [12].

4. The intersection of the three three-element sets is $\emptyset$. This case is investigated in Proposition 2.3.

\section{Results for $|X|=11$}

All the proofs in this Section follow a similar pattern: we assume that certain sets are in $\mathcal{F}$ and $\mathcal{F}$ is not Frankl's. Therefore, $\mathcal{F}$ contains no one- or two-element sets, and no case considered in the previous Lemmas occurs. Moreover, when considering the situation in a certain hypercube $\mathcal{C}$, unless otherwise stated, we are trying to prove that $s(\mathcal{C}) \geq d(\mathcal{C})$ and assuming the opposite.

Lemma 3.1. If $|X|=11$ and $\mathcal{F}$ contains two three-element sets with a two-element intersection, then $\mathcal{F}$ is Frankl's.

Proof. Let $\{a, b, c\}$ and $\{a, b, d\}$ be the two sets in $\mathcal{F}$. We consider the weight function $w$, with $w(a)=w(b)=8, w(c)=w(d)=6$ and $w(x)=1$ for $x \in X-\{a, b, c, d\}$. We have $t(w)=17.5$. Let $\mathcal{C}$ be an $\{a, b, c, d\}$-hypercube with bottom set $K$. We consider the cases:

1. $|K|=0$. Only four sets in this hypercube are in $\mathcal{F}$ (according to Proposition 2.1), so $s(\mathcal{C})=d(\mathcal{C})+2$.

2. $|K|=1$. In such hypercubes $p_{0}=p_{1}=0$, so the only sets which might have a deficit are on level 2. The surplus of the top set $K \cup\{a, b, c, d\}$ is 11.5 , and $d\left(\mathcal{C}_{2}\right) \geq 12$ implies $p_{2} \geq 4$. This means that $p_{3} \geq 3$, and $s(\mathcal{C}) \geq 24>d(\mathcal{C})$.

3. $|K|=2$. Here $d\left(\mathcal{C}_{2}\right) \leq 9.5$. If we consider the number of level 1 sets, we have subcases: 
(a) $p_{1}=0$. Then $s(\mathcal{C}) \geq 12.5>9.5 \geq d(\mathcal{C})$.

(b) $p_{1}=1$. That level 1 set implies that at least one of the sets $K \cup\{a, b, c\}$ and $K \cup\{a, b, d\}$ is in $\mathcal{F}$ (each has the surplus 6.5). Therefore, as the deficit of a level 1 set is at most $9.5, s(\mathcal{C}) \geq 19 \geq d(\mathcal{C})$.

(c) $p_{1}=2$. This implies that both of the sets $K \cup\{a, b, c\}$ and $K \cup\{a, b, d\}$ are in $\mathcal{F}$, so $s(\mathcal{C}) \geq 25.5$. Here $d\left(\mathcal{C}_{1}\right) \leq 19$, and this means that $s(\mathcal{C}) \geq d(\mathcal{C})$ provided that $d\left(\mathcal{C}_{2}\right) \leq 6.5$. But, $d\left(\mathcal{C}_{2}\right)>6.5$ implies $p_{2} \geq 4$ which implies $p_{3} \geq 3$, so $s(\mathcal{C}) \geq 30>28.5=19+9.5 \geq d\left(\mathcal{C}_{1}\right)+d\left(\mathcal{C}_{2}\right)=d(\mathcal{C})$.

(d) $p_{1} \geq 3$. Then these level 1 sets form three three-element sets with a common two-element intersection. Then $\mathcal{F}$ is Frankl's by Proposition 2.2.

4. $|K|=3$. If $K \notin \mathcal{F}$, the surplus of the top set is 13.5 . The sets producing a deficit are on level 1 (two with deficit 8.5 and two with deficit 6.5) and on level 2 (four with deficit 0.5 and one with deficit 2.5). Thus, $p_{1} \geq 2$, which implies that $K \cup\{a, b, c\}$ and $K \cup\{a, b, d\}$ are both in $\mathcal{F}$. So, $s(\mathcal{C}) \geq 28.5$, and therefore $p_{1}=4$. But then, $\mathcal{C} \backslash\{K\} \subseteq \mathcal{F}$, so $s(\mathcal{C})=41>34.5=d(\mathcal{C})$.

If $K \in \mathcal{F}$ we would like to prove that $d(\mathcal{C}) \leq s(\mathcal{C})+8$. The equality is achieved when $\mathcal{C} \subseteq \mathcal{F}$, which happens exactly when $p_{1}=4$. We consider the remaining cases for $p_{1}$. The deficit of $K$ is 14.5 , while $d\left(\mathcal{C}_{2}\right) \leq 4.5$. On the other hand, $s\left(\mathcal{C}_{4}\right)+s(K \cup\{a, b, c\})+s(K \cup\{a, b, d\})=28.5$, so $s(\mathcal{C})+8 \geq 36.5$. This means that $d\left(\mathcal{C}_{1}\right) \geq 18$, so $p_{1}=3$. We have, up to a trivial equivalence, two subcases: Either $K \cup\{a\} \notin \mathcal{F}$, or $K \cup\{c\} \notin \mathcal{F}$. In the first subcase, $p_{3} \geq 3$ and $s(\mathcal{C})+8 \geq 42$. Since $d\left(\mathcal{C}_{0}\right)+d\left(\mathcal{C}_{1}\right)=38$, we need $d\left(\mathcal{C}_{2}\right)=4.5$, so $p_{2} \geq 5$. But, this would imply $p_{3}=4$ and $s(\mathcal{C})+8 \geq 47.5>d(\mathcal{C})$. In the second subcase, we are guaranteed that $K \cup\{a, b\} \in \mathcal{F}$, so $s(\mathcal{C})+8 \geq 38$. Also, if $K \cup\{c, d\} \in \mathcal{F}$, then $p_{3}=4$, and the desired inequality trivially holds. The remaining case is when $d\left(\mathcal{C}_{2}\right) \leq 2$, so $d(\mathcal{C}) \leq 38 \leq s(\mathcal{C})+8$.

5. $|K|=4$. In this case and all others when $|K| \geq 4$ we only need to consider the case $K \in \mathcal{F}$ (so $K, K \cup\{a, b, c\}, K \cup\{a, b, d\}, K \cup\{a, b, c, d\} \in \mathcal{F})$, as otherwise we just imitate the proof for $|K|=3$, and the numbers work even better. We have that $s(\mathcal{C}) \geq 31.5$ and $d(\mathcal{C}) \geq 13.5$. Therefore, $d\left(\mathcal{C}_{1}\right)+d\left(\mathcal{C}_{2}\right)>18$. Hence, $p_{1} \geq 3$ and this means that either $K \cup\{a, b\} \in \mathcal{F}$, or $p_{3} \geq 3$. So, we now have $s(\mathcal{C}) \geq 34$ and either $p_{1}=4$ (in which case $\mathcal{C} \subseteq \mathcal{F}$ and the inequality $s(\mathcal{C}) \geq d(\mathcal{C})$ holds), or the only set with deficit which is not in $\mathcal{F}$ is one of the sets $K \cup\{a\}, K \cup\{b\}$. In the second case, we are forced to have $p_{3} \geq 3$ and $s(\mathcal{C})>35.5=d(\mathcal{C})$.

6. When $|K|=5, K \in \mathcal{F}$, we will prove that $s(\mathcal{C}) \geq d(\mathcal{C})+8.5$. We have $s(\mathcal{C}) \geq 34.5$ and $d(\mathcal{C})+8.5 \geq 21$. Again, the only sets with a deficit are the level 1 sets and $K \cup\{c, d\}$, and their weights guarantee that $p_{1} \geq 3$ (when $p_{1}=2$ only $s(\mathcal{C})=$ $d(\mathcal{C})+8.5$ is reachable). $p_{1} \geq 3$ means that $p_{2} \geq 3$, and $s\left(\mathcal{C}_{2}\right) \geq 3$, so $s(\mathcal{C}) \geq 37.5$. Therefore, $K \cup\{c\}, K \cup\{d\} \in \mathcal{F}$. In this case, we are forced to have $p_{3} \geq 3$ and $s(\mathcal{C}) \geq 46>43.5 \geq d(\mathcal{C})+8.5$. 
7. $6 \leq|K| \leq 7$ and $K \in \mathcal{F}$ are dealt with analogously to the case $|K|=5, K \in \mathcal{F}$. In both situations we obtain that the 'worst' case is when

$$
\begin{aligned}
& \mathcal{C} \cap \mathcal{F}=\{K, K \cup\{c\}, K \cup\{d\}, K \cup\{c, d\}, \\
& K \cup\{a, b, c\}, K \cup\{a, b, d\}, K \cup\{a, b, c, d\}\} .
\end{aligned}
$$

In case $|K|=6$ this implies that $s(\mathcal{C}) \geq d(\mathcal{C})+15.5$ and in the case $|K|=7$ this implies that $s(\mathcal{C}) \geq d(\mathcal{C})+22.5$.

8. $|K|=7$ and $K \notin \mathcal{F}$. We know that the top set is in $\mathcal{F}$, and if no other set is in $\mathcal{F}$, we have $s(\mathcal{C})=d(\mathcal{C})+17.5$. If $p_{1} \leq 2$, then $p_{1} \leq p_{3}$, and therefore $d(\mathcal{C})=d\left(\mathcal{C}_{1}\right) \leq s\left(\mathcal{C}_{3}\right)$. This means that we always have $s(\mathcal{C}) \geq d(\mathcal{C})+17.5$.

We have proved that the top and bottom hypercube together have the surplus by at least 19.5 greater than the deficit. Thus, there are at least 3 of the 'bad' hypercubes with $|K|=3, K \in \mathcal{F}$, in which $s(\mathcal{C}) \geq d(\mathcal{C})-8$. Consider the family of bottom sets of these hypercubes $\mathcal{G} \subseteq \mathcal{F}$. According to Theorem 2.1, $\mathcal{G}$ is either a 6 -set or a 7 -set. If $\bigcup \mathcal{G}$ is a 6 -set, then there are two of the bottom sets whose union is a 5-set. Therefore we have a hypercube with $|K|=6$ for which $s(\mathcal{C}) \geq d(\mathcal{C})+15.5$, and a hypercube with $|K|=5$ for which $s(\mathcal{C}) \geq d(\mathcal{C})+8.5$. The total surplus from the four 'good' hypercubes (the top one, the bottom one and the two we just established) is by at least 43.5 greater than the deficit. If $\bigcup \mathcal{G}$ is a 7 -set, then the difference between the total surplus and the total deficit of 'good' hypercubes is greater than 41.5 (we have that $K \in \mathcal{F}$ in the top hypercube, and also in at least two other ones with $|K| \geq 5$ ).

This means that $|\mathcal{G}| \geq 6$. Also, since no 5 -set contains more than two 3 -sets in $\mathcal{F}$, we get that any 6 -set can contain at most four 3 -sets in $\mathcal{F}$. We now know that the union of any six 3 -sets in $\mathcal{G}$ is $X-\{a, b, c, d\}$, and the surplus of the top and bottom hypercube must be by at least 24.5 greater than the deficit. An easy pigeon-hole argument shows that there must be at least four elements in $X-\{a, b, c, d\}$ which are 'covered' by at most three out of any six 3 -sets in $\mathcal{G}$, so the union of the remaining three (or more) must be a 6-set. This 6 -set is in $\mathcal{F}$, so we get four hypercubes with $|K|=6$ and $K \in \mathcal{F}$ for which $s(\mathcal{C}) \geq d(\mathcal{C})+15.5$. The total surplus of these four hypercubes, and the top and the bottom one, is by at least 86.5 greater than its total deficit. This means that $|\mathcal{G}| \geq 11$. But Theorem 2.1 and our inequality (1) imply $|\mathcal{G}| \leq 7$.

Lemma 3.2. If $|X|=11$ and $\mathcal{F}$ contains three four-element subsets of a five-element set, then $\mathcal{F}$ is Frankl's.

Proof. We suppose $\mathcal{F}$ is not Frankl's, so we may assume that $\mathcal{F}$ contains no one- or twoelement sets. Let $\{a, b, c, d\},\{a, b, c, e\},\{a, b, d, e\} \in \mathcal{F}$. We consider the weight function $w$, with $w(a)=w(b)=w(c)=w(d)=w(e)=4$, and $w(x)=1$ for $x \in X-\{a, b, c, d, e\}$. Then $t(w)=13$. Let $\mathcal{C}$ be an $\{a, b, c, d, e\}$-hypercube with bottom set $K$. We consider several cases, depending on $|K|$ : 
1. $|K|=0$. We know that $d(\emptyset)=13$ and according to Theorem $2.1, p_{3} \leq 2$, hence $d(\mathcal{C}) \leq 15$. On the other hand, $s(\mathcal{C})=s\left(\mathcal{C}_{4}\right)+s\left(\mathcal{C}_{5}\right) \geq 16$

2. $|K|=1$. In such hypercubes the top set has the surplus $8, d(\mathcal{C})=d\left(\mathcal{C}_{2}\right)$, but according to Lemma $3.1, p_{2} \leq 2$, so $d(\mathcal{C}) \leq 8$.

3. $|K|=2$. According to Lemma $3.1, p_{1} \leq 1$, so $d\left(\mathcal{C}_{1}\right) \leq 7$, while the surplus of the top set is 9 . Also, $d(\mathcal{C})=d\left(\mathcal{C}_{1}\right)+d\left(\mathcal{C}_{2}\right), d\left(\mathcal{C}_{2}\right)=3 p_{2}$, and $s\left(\mathcal{C}_{4}\right)=5 p_{4}$. The inequality $2 p_{4} \geq p_{2}$ follows by an easy case analysis. If $p_{2} \leq 5$, then $d\left(\mathcal{C}_{2}\right)-s\left(\mathcal{C}_{4}\right) \leq 2$ which gives $s(\mathcal{C}) \geq d(\mathcal{C})$. If $p_{2} \geq 6$, from inequality $(2)$ we get $3 p_{2} \leq 2 p_{3}+10$ and $p_{3} \geq 4$. Thus we have $s(\mathcal{C})-d(\mathcal{C})=s\left(\mathcal{C}_{4}\right)-d\left(\mathcal{C}_{2}\right)+s\left(\mathcal{C}_{5}\right)-d\left(\mathcal{C}_{1}\right)+s\left(\mathcal{C}_{3}\right) \geq-5+2+4=1$.

4. $|K|=3$. If $K \notin \mathcal{F}$, the surplus of the top set is 10 , the surplus of a level 4 set is equal to the deficit of a level 1 set (both 6), and the surplus of a level 3 set is equal to the deficit of a level 2 set (both 2 ). If $p_{1}>p_{4}$, then $p_{1}=4$ and $p_{4}=3$. Now we have $p_{3} \geq 4$, which together with (2) gives $p_{2} \leq p_{3}+2$. Clearly, in this case $s(\mathcal{C}) \geq d(\mathcal{C})$. If $p_{1} \leq p_{4}$, using $p_{2} \leq p_{3}+3$ (which is a consequence of $(2)$ ), we conclude that $s(\mathcal{C}) \geq d(\mathcal{C})+4$ holds.

If $K \in \mathcal{F}$, such hypercubes may have a deficit. We can see from the previous case that $d(\mathcal{C}) \leq s(\mathcal{C})+10$ and there are examples of hypercubes in which equality holds.

5. $|K|=4$. The surplus of the top set is 11 , the deficit of the bottom set is 9 . If there are at least one set on levels lower than 3 , then $s\left(\mathcal{C}_{4}\right)=7 p_{4} \geq 5 p_{1}+1=d\left(\mathcal{C}_{2}\right)+1$. As the deficit of a level 2 set is 1 , inequality (2) implies $d\left(\mathcal{C}_{2}\right) \leq s\left(\mathcal{C}_{3}\right)+3$. This implies $s(\mathcal{C}) \geq d(\mathcal{C})$.

6. $|K|=5$. We will only examine the case $K \in \mathcal{F}$ and try to prove $s(\mathcal{C}) \geq d(\mathcal{C})+20$. In this situation, $s\left(\mathcal{C}_{4}\right)+s\left(\mathcal{C}_{5}\right) \geq 36$. $K$ has the deficit 8 ; levels 1 and 3 have equal deficit/surplus, and level 2 sets have weight $t(w)$. From $p_{1} \leq p_{3}+2$ follows that $d\left(\mathcal{C}_{1}\right)-s\left(\mathcal{C}_{3}\right) \leq 8$ and $s(\mathcal{C}) \geq d(\mathcal{C})+20$.

7. $|K|=6$. The surplus of the top set is 13 . If $K \notin \mathcal{F}, d\left(\mathcal{C}_{1}\right)=3 p_{1} \leq 9 p_{4}=s\left(\mathcal{C}_{4}\right)$, so $s(\mathcal{C}) \geq d(\mathcal{C})+13$. If $K \in \mathcal{F}$, then using similar arguments as in the case $|K|=5$, we can prove $s(\mathcal{C}) \geq d(\mathcal{C})+28$.

We have proved that in the top hypercube $s(\mathcal{C}) \geq d(\mathcal{C})+13$ holds. Thus, there are at least two of the 'bad' hypercubes with $|K|=3, K \in \mathcal{F}$, in which $s(\mathcal{C}) \geq d(\mathcal{C})-10$. Consider the family of bottom sets of these hypercubes $\mathcal{G} \subseteq \mathcal{F}$. Lemma 3.1 guarantees $|\mathcal{G}| \leq 4$. If $|\mathcal{G}|=2$ then, according to Lemma $3.1,5 \leq|\bigcup \mathcal{G}| \leq 6$ and $\bigcup \mathcal{G} \in \mathcal{F}$ is the bottom set of a hypercube $\mathcal{C}$. In both cases, $s(\mathcal{C}) \geq d(\mathcal{C})+20$. If $|\mathcal{G}| \geq 3$, then the surplus of the top hypercube is by at least 28 greater than its deficit, and there will be a hypercube $\mathcal{C}$ with $|K|=5$ and $s(\mathcal{C}) \geq d(\mathcal{C})+20$. Thus $\mathcal{F}$ is Frankl's.

Lemma 3.3. Let $|X|=11$ and $\mathcal{F}$ contains three four-element sets which all contain the same three elements. Then $\mathcal{F}$ is Frankl's. 
Proof. Let $\{a, b, c, d\},\{a, b, c, e\},,\{a, b, c, f\} \in \mathcal{F}$. The weight function we choose is $w(x)=3$ for $x \in\{a, b, c, d, e, f\}$ and $w(x)=1$ for all other $x \in X$. The target weight is 11.5. We consider an $\{a, b, c, d, e, f\}$-hypercube $\mathcal{C}$ with bottom set $K$. Again we have several possible cases depending on $|K|$.

1. $|K|=0$. Here $p_{4} \geq 3$ and $p_{5} \geq 3$, so $s(\mathcal{C}) \geq 18.5$, while $d(\emptyset)=11.5$. Lemma 3.1 implies $p_{3} \leq 4$, so $d\left(\mathcal{C}_{3}\right) \leq 10$. If $p_{3} \geq 3$ then we must have that any two level 3 sets intersect (according to Lemma 3.1), so $p_{3}=4$ implies $p_{5}=6$ and $s(\mathcal{C})>d(\mathcal{C})$. The worst case is $p_{3}=3$ when $s(\mathcal{C})+0.5 \geq d(\mathcal{C})$.

2. $|K|=1$. Then the surplus of the top set of $\mathcal{C}$ is 7.5 and $p_{0}=p_{1}=0$. Since $p_{3} \leq p_{4}+5$ (according to $\left.(2)\right), d\left(\mathcal{C}_{3}\right)=1.5 p_{3} \leq 1.5 p_{4}+7.5=s\left(\mathcal{C}_{4}\right)+7.5$. By Lemma 3.1 , the intersection of any two level 2 sets is $K$, so $p_{2} \leq 3$. Hence, $p_{2} \leq p_{5}$ and $d\left(\mathcal{C}_{2}\right)=4.5 p_{2} \leq 4.5 p_{5}=s\left(\mathcal{C}_{5}\right)$, so $d(\mathcal{C}) \leq s(\mathcal{C})$.

3. $|K|=2$. Then $K \notin \mathcal{F}$, and by Lemma $3.1, p_{1} \leq 1$. The surplus of the top set is 8.5 . By Lemma 3.2 and Turan's Theorem, each level $k$ set which is in $\mathcal{F}$ can contain at most $\frac{k^{2}}{4}$ level 2 sets which are in $\mathcal{F}$. From Lemma 2.3 we conclude $3 p_{2} \leq p_{4}+15$ and $p_{3} \leq p_{4}+5$. The second inequality implies $d\left(\mathcal{C}_{3}\right) \leq s\left(\mathcal{C}_{4}\right)+2.5$, so $p_{1}=1$ or $p_{2} \geq 2$. Both imply $p_{5} \geq 2$, so $s\left(\mathcal{C}_{5}\right)+s\left(\mathcal{C}_{6}\right) \geq 19.5, d\left(\mathcal{C}_{1}\right)+d\left(\mathcal{C}_{2}\right)>17$ and $d\left(\mathcal{C}_{2}\right) \geq 11$.

If $p_{5}=2$, then $p_{2} \leq 5$ (otherwise all three level 5 sets containing $\{a, b, c\}$ would be in $\mathcal{F})$. Let $K \cup\{a, b, c, d, e\} \notin \mathcal{F}$. Then all level 2 sets in $\mathcal{F}$ are in $\mathcal{C}_{1}^{f}$, and $d\left(\mathcal{C}_{2}\right) \geq 11$ implies $p_{2}=p_{1}\left(\mathcal{C}^{f}\right) \geq 4$, so $p_{3}\left(\mathcal{C}^{f}\right) \geq 4$. Therefore, $p_{4} \geq 4$, and $p_{3} \leq p_{4}+5$ implies $d\left(\mathcal{C}_{3}\right) \leq s\left(\mathcal{C}_{4}\right)-5.5$. Now, $d\left(\mathcal{C}_{1}\right)+d\left(\mathcal{C}_{2}\right)>25$, so $p_{2} \geq 6$, which is a contradiction.

If $p_{5} \geq 3$, then $s(\mathcal{C})-d\left(\mathcal{C}_{3}\right) \geq 22.5$. Therefore, $p_{2} \geq 5$ and $p_{4} \geq 1$ (at least one of the level 4 sets containing $\{a, b, c\}$ must be in $\mathcal{F}$ when $\left.p_{2}>3\right)$. Let $p_{4}=3 l+s$, $0 \leq s \leq 2$. Then $p_{3} \leq 3 l+s+5$, while $3 p_{2} \leq p_{4}+15$ implies $p_{2} \leq l+5$. Therefore, $d\left(\mathcal{C}_{2}\right)+d\left(\mathcal{C}_{3}\right)-s\left(\mathcal{C}_{4}\right) \leq 20-2.5 l-2 s \leq 18$. On the other hand, $s\left(\mathcal{C}_{5}\right)+s\left(\mathcal{C}_{6}\right)-d\left(\mathcal{C}_{1}\right) \geq$ 18.5, so $s(\mathcal{C}) \geq d(\mathcal{C})$.

4. $|K|=3$ and $K \notin \mathcal{F}$. There are four cases depending on $p_{1}$.

(a) $p_{1} \leq 3$. Then $p_{4} \geq p_{1}$ and $d\left(\mathcal{C}_{1}\right)-s\left(\mathcal{C}_{4}\right)=5.5 p_{1}-3.5 p_{4} \leq 6$. The surplus of the top set is 9.5 , which means that $d\left(\mathcal{C}_{2}\right)=2.5 p_{2}>3.5$, so $p_{2} \geq 2$. This implies $p_{5} \geq 2$, and as $s\left(\mathcal{C}_{5}\right)=6.5 p_{5} \geq 13$, we have $p_{2} \geq 7$. Now $p_{5} \geq 3$, and $d\left(\mathcal{C}_{2}\right)>23$. This gives $p_{2} \geq 10$. From inequality (2) we get $2 p_{2} \leq p_{3}+10$. Now we have $p_{3} \geq 10$, and from $s\left(\mathcal{C}_{3}\right)=0.5 p_{3}$, we obtain $p_{2} \leq 12$. Now it is easy to verify that $p_{5}=6$ and $s(\mathcal{C}) \geq d(\mathcal{C})$.

(b) $p_{1}=4$. From $d\left(\mathcal{C}_{1}\right)=22, p_{4} \geq 3$ and $p_{5} \geq 3$ we conclude $d\left(\mathcal{C}_{2}\right)>17.5$ and $p_{2} \geq 8$. If one considers the level 2 sets which have one-element intersection with $\{a, b, c\}$, and the remaining ones, it is easy to see that at least 4 in any of those groups produce a level 4 set not containing $\{a, b, c\}$, so $p_{4} \geq 4$. This gives $p_{2} \geq 9$ and (by Lemma 2.3) $p_{3} \geq 8$. Now $s(\mathcal{C}) \geq 47$, which gives $p_{2} \geq 11$. Now for at most one $x \in\{a, b, c, d, e, f\}, p_{2}\left(\mathcal{C}^{\neg x}\right) \leq 6$, and the top set of $\mathcal{C}^{\neg x}$ is not in $\mathcal{F}$. So, $p_{5} \geq 5$ which implies $s(\mathcal{C}) \geq d(\mathcal{C})$. 
(c) $p_{1}=5$. Let $K \cup\{x\}$ be the level 1 set not in $\mathcal{F}$. Then all five level 4 sets not containing $x$ are in $\mathcal{F}$ and at least one of the sets $K \cup\{a, b, c, d\}, K \cup\{a, b, c, e\}$, $K \cup\{a, b, c, f\}$ which contains $x$ is in $\mathcal{F}$. So $p_{4} \geq 6, p_{3} \geq 10$ and $p_{5} \geq 3$ holds. This means that $s(\mathcal{C}) \geq 55$, which gives $p_{2} \geq 12$. Now it is easy to verify $p_{5}=6$ which implies $s(\mathcal{C}) \geq d(\mathcal{C})$.

(d) $p_{1}=6$. Then all the sets from $\mathcal{C}$ except $K$ are in $\mathcal{F}$.

If $K \in \mathcal{F}$ then, clearly, $s(\mathcal{C})+8.5 \geq d(\mathcal{C})$.

5. $|K|=4$. If $K \notin \mathcal{F}$, we can imitate the proof for $|K|=3$. If $K \in F, 2 p_{2} \leq p_{3}+10$ implies $d\left(\mathcal{C}_{2}\right)-s\left(\mathcal{C}_{3}\right) \leq 7.5 . s\left(\mathcal{C}_{4}\right)+s\left(\mathcal{C}_{5}\right)+s\left(\mathcal{C}_{6}\right) \geq 46.5$, while $d\left(\mathcal{C}_{0}\right)+d\left(\mathcal{C}_{1}\right) \leq 34.5$, so $s(\mathcal{C}) \leq d(\mathcal{C})$.

6. $|K|=5$. Here $2 p_{2} \leq p_{3}+10$ implies $d\left(\mathcal{C}_{2}\right)-s\left(\mathcal{C}_{3}\right) \leq 2.5$. If $K \in F$, then $s\left(\mathcal{C}_{4}\right)+$ $s\left(\mathcal{C}_{5}\right)+s\left(\mathcal{C}_{6}\right) \geq 53.5$ and $d\left(\mathcal{C}_{0}\right)+d\left(\mathcal{C}_{1}\right) \leq 27.5$. This gives $s(\mathcal{C}) \geq d(\mathcal{C})+23.5$. If $K \notin F$ it is easy to see that if $d(\mathcal{C})>0$, then $p_{5}>0$, and (quite straightforwardly) $d(\mathcal{C}) \leq s\left(\mathcal{C}_{5}\right)$. The 'worst' case is $d(\mathcal{C})=0$ when $s(\mathcal{C}) \geq d(\mathcal{C})+11.5$.

We have proved that, except for the bottom hypercube with possible deficit 0.5, 'bad' hypercubes can appear only at level 3 and there $d(\mathcal{C}) \leq s(\mathcal{C})+8.5$. According to Lemma 3.1, there can be at most two of them. If there is only one, its extra deficit is covered by the top hypercube. If there are two of them, according to Lemma 3.1, in the top hypercube it holds $K \in F$, so the top hypercube satisfies $s(\mathcal{C}) \geq d(\mathcal{C})+23.5$ and easily makes up for all extra deficit.

Lemma 3.4. Let $|X|=11$ and $\mathcal{F}$ contains two four-element subsets of a five element set. Than $\mathcal{F}$ is Frankl's.

Proof. Let $\{a, b, c, d\},\{a, b, c, e\} \in \mathcal{F}$. We choose the weight function such that $w(a)=$ $w(b)=w(c)=w(d)=w(e)=4$ and $w(x)=1$ for all other $x \in X$, so $t(w)=13$. Again we observe an $\{a, b, c, d, e\}$-hypercube $\mathcal{C}$ with bottom set $K$ and consider cases:

1. $|K|=0$. Here $d(\emptyset)=13$ and by Lemma 3.1, $p_{3} \leq 2$ and $d\left(\mathcal{C}_{3}\right) \leq 2$. On the other hand, $s(\mathcal{C}) \geq 13$, so $s(\mathcal{C})+2 \geq d(\mathcal{C})$.

2. $|K|=1$. Here $d(\mathcal{C})=d\left(\mathcal{C}_{2}\right)=4 p_{2}$. By Lemma $3.1, p_{2} \leq 2$ and $d(\mathcal{C}) \leq 8$. The surplus of the top set is 8 , so $s(\mathcal{C}) \geq d(\mathcal{C})$.

3. $|K|=2$. Lemma 3.1 gives $p_{1} \leq 1$. By Lemma 3.3 every element from $\{a, b, c, d, e\}$ can appear in at most two level 2 sets from $\mathcal{F}$. This implies $p_{2} \leq 5$. Also, from Lemma 3.2 and Lemma 2.3 we get $d\left(\mathcal{C}_{2}\right)=3 p_{2} \leq p_{3}+10=s\left(\mathcal{C}_{3}\right)+10$. If $p_{4} \geq 2$, then $s\left(\mathcal{C}_{4}\right)+s\left(\mathcal{C}_{5}\right) \geq 19>17 \geq d\left(\mathcal{C}_{1}\right)+d\left(\mathcal{C}_{2}\right)-s\left(\mathcal{C}_{3}\right)$. If $p_{4}=1$ then $\mathcal{F} \cap \mathcal{C}_{2} \subseteq \mathcal{C}_{1}^{d}$ or $\mathcal{F} \cap \mathcal{C}_{2} \subseteq \mathcal{C}_{1}^{e}$. Either way, $p_{2} \leq 2$ because $d$ (or $e$ ) can be in at most two level 2 sets, so $s(\mathcal{C}) \geq 14>13 \geq d(\mathcal{C})$. Finally, if $p_{4}=0$ then $p_{1}=0$ and $p_{2} \leq 1$, so $s(\mathcal{C}) \geq 9>3 \geq d(\mathcal{C})$. 
4. $|K|=3$. Let $K \notin \mathcal{F}$. From Lemma 3.3 it follows $p_{1} \leq 2$, and this implies $p_{1} \leq p_{4}$ and $d\left(\mathcal{C}_{1}\right)=6 p_{1} \leq 6 p_{4}=s\left(\mathcal{C}_{4}\right)$. By inequality (2) we get $3 p_{2} \leq 2 p_{3}+10$. Therefore, $d\left(\mathcal{C}_{2}\right)-s\left(\mathcal{C}_{3}\right)=2 p_{2}-2 p_{3} \leq 6$, while the surplus of the top set is 10 . When $K \in \mathcal{F}$ we will prove that $d(\mathcal{C}) \leq s(\mathcal{C})+4$. If $p_{1}<2$, according to above observations, we have $s(\mathcal{C}) \geq d(\mathcal{C})$. So we may assume $p_{1}=2$. If $p_{2} \geq 5$ it follows from $3 p_{2} \leq 2 p_{3}+10$ that $p_{2} \leq p_{3}+2$. If $p_{2}=3$ then $p_{3} \geq 1$. Finally, when $p_{2}=4$, either for an element $x \in\{a, b, c, d, e\}, p_{1}\left(\mathcal{C}^{x}\right) \geq 3$, and therefore $p_{3} \geq p_{2}\left(\mathcal{C}^{x}\right) \geq 3$ holds, or for any element $x \in\{a, b, c, d, e\}$ such that $K \cup\{x\} \in \mathcal{F}, p_{2}\left(\mathcal{C}^{\neg x}\right) \geq 2$, so the unions of these sets in $\mathcal{F} \cap \mathcal{C}_{2}^{\neg x}$ with $K \cup\{x\}$ give $p_{3} \geq p_{2}\left(\mathcal{C}^{x}\right) \geq 2$. In all cases for $p_{2}$, the inequality $p_{2} \leq p_{3}+2$ holds. Then $d\left(\mathcal{C}_{2}\right) \leq s\left(\mathcal{C}_{3}\right)+4$ and $d\left(\mathcal{C}_{0}\right)+d\left(\mathcal{C}_{1}\right) \leq s\left(\mathcal{C}_{4}\right)+s\left(\mathcal{C}_{5}\right)$, which gives $s(\mathcal{C})+4 \geq d(\mathcal{C})$.

5. $4 \leq|K| \leq 5$ (we only consider the 'harder' case $|K|=4$ ). From $3 p_{2} \leq 2 p_{3}+10$ we conclude $p_{2} \leq p_{3}+3$ and $d\left(\mathcal{C}_{2}\right)=p_{2} \leq 3 p_{3}+3=s\left(\mathcal{C}_{3}\right)+3$. Since the surplus of the top set is 11 , either $K \in \mathcal{F}$, or $p_{1} \geq 2$. Either way, $p_{4} \geq 2$, and $s\left(\mathcal{C}_{4}\right)+s\left(\mathcal{C}_{5}\right) \geq 25$. Therefore, $d\left(\mathcal{C}_{1}\right)>13$, so $p_{1} \geq 3$. But, in this case, $p_{3} \geq 1$, so $d\left(\mathcal{C}_{2}\right) \leq s\left(\mathcal{C}_{3}\right)+1$. This implies $d\left(\mathcal{C}_{1}\right)>15$ and $p_{1} \geq 4$. Now $p_{3} \geq 4$ and $d\left(\mathcal{C}_{2}\right) \leq s\left(\mathcal{C}_{3}\right)-5$. This implies $d\left(\mathcal{C}_{1}\right)>21$ and $p_{1}=5$, but then $\mathcal{C} \backslash\{K\} \subseteq \mathcal{F}$ and $s(\mathcal{C}) \geq \bar{d}(\mathcal{C})+16$.

6. $|K|=6$. If $K \notin \mathcal{F}$, we will prove that $s(\mathcal{C}) \geq d(\mathcal{C})+13$. The surplus of the top set is 13 , while $d(\mathcal{C})=d\left(\mathcal{C}_{1}\right)=3 p_{1}$. But, if $p_{1} \leq 2$, then $p_{1} \leq p_{4}$ and if $p_{1}>2$, then $p_{4} \geq 2$. In both cases, $s\left(\mathcal{C}_{4}\right)=9 p_{4} \geq 3 p_{1}=d\left(\mathcal{C}_{1}\right)$. If $K \in \mathcal{F}$, we will prove that $s(\mathcal{C}) \geq d(\mathcal{C})+19$. We have $s\left(\mathcal{C}_{4}\right)+s\left(\mathcal{C}_{5}\right) \geq 31$ and $d\left(\mathcal{C}_{0}\right)+19=26$. Therefore, $p_{1} \geq 2$, and hence $p_{2} \geq 1$. Now $s(\mathcal{C}) \geq 32$ and $p_{1} \geq 3$. We have $p_{1} \leq p_{3}+2$, so $d\left(\mathcal{C}_{1}\right)-s\left(\mathcal{C}_{3}\right)=3 p_{1}-5 p_{3} \leq 6-2 p_{3} \leq 4$ and the claim follows.

We have the bottom hypercube with $d(\mathcal{C}) \leq s(\mathcal{C})+2$ and the top hypercube with $d(\mathcal{C}) \leq s(\mathcal{C})-13$. The only other hypercubes with $d(\mathcal{C})>s(\mathcal{C})$ are those with $|K|=3$, $K \in \mathcal{F}(d(\mathcal{C}) \leq s(\mathcal{C})+4)$. According to Lemma 3.1, we can have at most four such hypercubes in $\mathcal{F}$. However, from the extra surplus in the top hypercube we conclude we have at least three such 'bad' hypercubes in $\mathcal{F}$. By Lemma 3.1, this means that the union of their bottom sets must be $X-\{a, b, c, d, e\}$. Therefore, in the top hypercube, $K \in \mathcal{F}$ and $d(\mathcal{C}) \leq s(\mathcal{C})-19$. This cannot be 'covered' by the extra deficits of the bottom and 'bad' hypercubes.

Lemma 3.5. Let $|X|=11$ and $\mathcal{F}$ contain two intersecting three-element sets. Then $\mathcal{F}$ is Frankl's.

Proof. Let $\{a, b, c\},\{a, d, e\} \in \mathcal{F}$. The weight function we choose is $w(a)=8, w(b)=$ $w(c)=w(d)=w(e)=4$ and $w(x)=1$ for all other $x \in X$. The target weight $t(w)=15$. We consider an $\{a, b, c, d, e\}$-hypercube $\mathcal{C}$ with bottom set $K$. As usual, we consider possible values of $|K|$ :

1. $|K|=1$. Here, $p_{0}=p_{1}=0$ and according to Lemma 3.1, $p_{2} \leq 2$. Also, Lemma 3.4 implies that $p_{3} \leq 2$, and if $p_{3}=2$, then $p_{2}\left(\mathcal{C}^{a}\right) \geq 1$, so $d\left(\mathcal{C}_{3}\right) \leq 2$. Since 
$p_{2} \leq 2, p_{2} \leq p_{3}\left(\mathcal{C}^{a}\right)+p_{2}\left(\mathcal{C}^{a}\right)$. Therefore, $d\left(\mathcal{C}_{2}\right) \leq 6 p_{2} \leq 2 p_{3}\left(\mathcal{C}^{a}\right)+2 p_{2}\left(\mathcal{C}^{a}\right)+8 \leq$ $s\left(\mathcal{C}_{3}\right)+s\left(\mathcal{C}_{4}\right)+8=s(\mathcal{C})-2$.

2. $|K|=2$. Then $K \notin \mathcal{F}$, by Lemma $3.1, p_{1} \leq 1$, while by Lemma $3.4, p_{2} \leq 2$. The surplus of the top set is 11 and $d\left(\mathcal{C}_{3}\right) \leq 4$. If $d\left(\mathcal{C}_{1}\right)<9$, then $d\left(\mathcal{C}_{1}\right) \leq s\left(\mathcal{C}_{3}\right)$. This means that $d\left(\mathcal{C}_{2}\right)>7$, so $p_{2}=2$ and $d\left(\mathcal{C}_{2}\right)=10$. But, $p_{2}=2$ implies $p_{4} \geq 1$, and $s\left(\mathcal{C}_{4}\right)+s\left(\mathcal{C}_{5}\right) \geq 14 \geq d\left(\mathcal{C}_{2}\right)+d\left(\mathcal{C}_{3}\right)$, so $s(\mathcal{C}) \geq d(\mathcal{C})$.

Let us now assume that $p_{1}=1$ and $d\left(\mathcal{C}_{1}\right)=9$. This means that $p_{4} \geq 1, p_{3} \geq 1$, $s\left(\mathcal{C}_{4}\right) \geq 7$ and $s\left(\mathcal{C}_{3}\right) \geq 3$. Therefore $s(\mathcal{C}) \geq 21$, so $p_{2}=2$ and $d\left(\mathcal{C}_{2}\right)=10$. But then $K \cup\{b, c, d, e\} \in \mathcal{F}$, according to Lemma 3.4. Therefore, $s(\mathcal{C}) \geq 24>d(\mathcal{C})$.

3. $|K|=3$. We have $p_{1} \leq 1$, according to Lemma 3.4. The subfamily of $\mathcal{C}$

$$
\begin{gathered}
\mathcal{C}^{\prime}=\{K, K \cup\{a\}, K \cup\{b, c\}, K \cup\{d, e\}, K \cup\{a, b, c\}, \\
K \cup\{a, d, e\}, K \cup\{b, c, d, e\}, K \cup\{a, b, c, d, e\}\}
\end{gathered}
$$

has $s\left(\mathcal{C}^{\prime} \cap \mathcal{F}\right) \geq d\left(\mathcal{C}^{\prime} \cap \mathcal{F}\right)$. Let $q_{2}=\left|\mathcal{C}_{2} \cap\left(\mathcal{C}^{a} \backslash \mathcal{C}^{\prime}\right) \cap \mathcal{F}\right|$. If $q_{2}=0$, then $d((\mathcal{C} \backslash$ $\left.\left.\mathcal{C}^{\prime}\right) \cap \mathcal{F}\right)=8 p_{1}\left(\mathcal{C}_{1}^{\neg a}\right) \leq 8 p_{3}\left(\mathcal{C}^{a}\right) \leq s\left(\left(\mathcal{C} \backslash \mathcal{C}^{\prime}\right) \cap \mathcal{F}\right)$. If $q_{2}>0$, then $q_{2} \leq p_{3}\left(\mathcal{C}^{a}\right)-1$ or $q_{2}=p_{3}\left(\mathcal{C}^{a}\right)=4$. Hence, $d\left(\left(\mathcal{C} \backslash \mathcal{C}^{\prime}\right) \cap \mathcal{F}\right) \leq 4 q_{2}+8 \leq 8 p_{3}\left(\mathcal{C}^{a}\right) \leq s\left(\left(\mathcal{C} \backslash \mathcal{C}^{\prime}\right) \cap \mathcal{F}\right)$. Either way, $s(\mathcal{C})=s\left(\left(\mathcal{C} \backslash \mathcal{C}^{\prime}\right) \cap \mathcal{F}\right)+s\left(\mathcal{C}^{\prime} \cap \mathcal{F}\right) \geq d\left(\left(\mathcal{C} \backslash \mathcal{C}^{\prime}\right) \cap \mathcal{F}\right)+d\left(\mathcal{C}^{\prime} \cap \mathcal{F}\right)=d(\mathcal{C})$.

4. $4 \leq|K| \leq 5$. We will consider only the case $|K|=4$. Analogously to the case $|K|=3$, we define the subfamily $\mathcal{C}^{\prime} \subseteq \mathcal{C}$ and see easily that $s\left(\mathcal{C}^{\prime} \cap \mathcal{F}\right) \geq d\left(\mathcal{C}^{\prime} \cap \mathcal{F}\right)+4$. We have only eight sets with a deficit in $\mathcal{C} \backslash \mathcal{C}^{\prime}$, and can divide them among four groups of the form $K \cup\{b\}, K \cup\{b, d\}, K \cup\{a, b, d, e\}$. The total deficit of such a group is by at most 1 greater than the surplus. Therefore, $s(\mathcal{C}) \geq d(\mathcal{C})$.

5. $|K|=6$ and $|K|=0$. For $|K|=0$, according to Lemma 3.1, $d(\mathcal{C}) \leq s(\mathcal{C})+4$. But, considering that the weight of a set in the top hypercube is by 2 greater than of the corresponding set in the hypercube with $|K|=4$, if $|\mathcal{C} \cap \mathcal{F}| \geq 2$ in the top hypercube, the proof for $|K|=4$ implies that $s(\mathcal{C}) \geq d(\mathcal{C})+4$ for $|K|=6$. If $|\mathcal{C} \cap \mathcal{F}|=1$ in the top hypercube, then $\mathcal{C} \cap \mathcal{F}=\{X\}$, so $s(\mathcal{C}) \geq d(\mathcal{C})+4$, again.

Lemma 3.6. Let $|X|=11$ and $\mathcal{F}$ contain two three-element sets. Then $\mathcal{F}$ is Frankl's.

Proof. Let $\{a, b, c\},\{d, e, f\} \in \mathcal{F}$. The weight function we choose is $w(x)=2.5$ for $x \in\{a, b, c, d, e, f\}$ and $w(x)=1$ for all other $x \in X$. The target weight is 10 . We consider an $\{a, b, c, d, e, f\}$-hypercube $\mathcal{C}$ with bottom set $K$. We will prove that the only hypercube with $d(\mathcal{C})-s(\mathcal{C})>0$ is the bottom one, and that this difference will be covered by the extra surplus in the top hypercube.

1. $|K|=0$. Since $p_{3}=2, d(\mathcal{C})-s(\mathcal{C}) \leq 10+2.5 p_{3}-5 \leq 10$.

2. $|K|=1$. We have $p_{0}=p_{1}=p_{2}=0$. According to Lemma $3.4, p_{3} \leq 4$, and therefore $s(\mathcal{C}) \geq s\left(\mathcal{C}_{6}\right)=6 \geq 1.5 p_{3}=d(\mathcal{C})$. 
3. $|K|=2$. From Lemma 3.5 we get $p_{1}=0$, from Lemma 3.4 we get $p_{2} \leq 3$, while $d\left(\mathcal{C}_{3}\right)=0.5 p_{3}$. All sets in $\mathcal{C}_{3} \cup \mathcal{C}_{5}$, except $K \cup\{a, b, c\}$ and $K \cup\{d, e, f\}$, can be divided into six groups of the form $K \cup\{a, b, d\}, K \cup\{a, b, e\}, K \cup\{a, b, f\}$, $K \cup\{a, b, d, e, f\}$. The total deficit of such a group is not greater than the surplus, so we have $d\left(\mathcal{C}_{3}\right)-s\left(\mathcal{C}_{5}\right) \leq 1$. The surplus of the top set is 7 . This gives $d\left(\mathcal{C}_{2}\right)=3 p_{2}>6$ and $p_{2}=3$. By Lemma 3.4 this forces $p_{4} \geq 3$, which means $s(\mathcal{C}) \geq d(\mathcal{C})$.

4. $|K|=3$. If $p_{4}+p_{5}=0$, then $d(\mathcal{C}) \leq d(K)=7<8 \leq s(\mathcal{C})$. So we may assume $p_{4}+p_{5}>0$. From Lemma 3.4 we conclude $p_{1} \leq 1$. Also, we can notice that $s\left(\mathcal{C}_{3}\right)=0.5 p_{3} \geq p_{0}$. Let $\mathcal{Q}=\left\{Y \in \mathcal{C}_{2}:|Y \cap\{a, b, c\}|=1\right\}, \mathcal{R}=\mathcal{C}_{2} \backslash \mathcal{Q}, q_{2}=|\mathcal{Q} \cap \mathcal{F}|$ and $r_{2}=|\mathcal{R} \cap \mathcal{F}|$. Since $r_{2} \leq p_{5}, s\left(\mathcal{C}_{5}\right)-2 r_{2}=5.5 p_{5}-2 r_{2} \geq 3.5 p_{5}$. Consider the bipartite graph $G=(V, E)$ where $V=\{a, b, c, d, e, f\}$ and $x y \in E$ iff $K \cup\{x, y\} \in \mathcal{Q}$. Let $m=\left|\left\{x \in\{a, b, c\}: d_{G}(x)>0\right\}\right|$ and $n=\left|\left\{x \in\{d, e, f\}: d_{G}(x)>0\right\}\right|$. Then $q_{2} \leq m n \leq m+n+\left(\begin{array}{c}m \\ 2\end{array}\right)+\left(\begin{array}{c}n \\ 2\end{array}\right)-1 \leq p_{4}+p_{5}-1$. Now $s(\mathcal{C})-d(\mathcal{C}) \geq s\left(\mathcal{C}_{6}\right)+s\left(\mathcal{C}_{5}\right)+$ $3 p_{4}+p_{0}-2 q_{2}-2 r_{2}-4.5 p_{1}-7 p_{0} \geq 8+3-4.5-6 p_{0}+3.5 p_{5}+3\left(p_{4}-1\right)-2 q_{2} \geq$ $0.5+2\left(p_{5}+p_{4}-1-q_{2}\right) \geq 0$.

5. $|K|=4$. Here $s\left(\mathcal{C}_{3}\right)=1.5 p_{3} \geq 3 p_{0}$, so $s\left(\mathcal{C}_{6}\right)+s\left(\mathcal{C}_{3}\right)-d\left(\mathcal{C}_{0}\right) \geq 9-3 p_{0} \geq 6$. Since $p_{1} \leq p_{4}, s\left(\mathcal{C}_{4}\right)-d\left(\mathcal{C}_{1}\right)=4 p_{4}-3.5 p_{1} \geq 0$. Since $d\left(\mathcal{C}_{2}\right)=p_{2}$, we have $p_{2} \geq 7$. But now, using a similar argument with $q_{2}$ and $r_{2}$ as above, we get $p_{5} \geq 2$, which implies $15 \geq p_{2}=d\left(\mathcal{C}_{2}\right)>19$, a contradiction.

6. $|K|=5$. Since $p_{1} \leq p_{4}$ and $s\left(\mathcal{C}_{3}\right)=2.5 p_{3} \geq 5 p_{0}$, we get $s(\mathcal{C})-d(\mathcal{C}) \geq 10+7.5 p_{5}+$ $5 p_{4}+5 p_{0}-5 p_{1}-5 p_{0} \geq 10$.

Lemma 3.7. Let $|X|=11$ and $\mathcal{F}$ contain a four-element set and one of its three-element subsets. Then $\mathcal{F}$ is Frankl's.

Proof. Let $\{a, b, c\},\{a, b, c, d\} \in \mathcal{F}$. The weight function will be $w(a)=w(b)=w(c)=3$, $w(d)=2$ and $w(x)=1$ for all other $x \in X$. The target weight is 9 . Let $\mathcal{C}$ be an $\{a, b, c, d$,$\} -hypercube with bottom set K$. We consider the cases:

1. $|K|=1$. Here $d(\mathcal{C})=0$.

2. $|K|=2$. By Lemma 3.4 we get $p_{2} \leq 2$, which gives $s(\mathcal{C}) \geq s\left(\mathcal{C}_{4}\right) \geq 4 \geq 2 p_{2} \geq d(\mathcal{C})$.

3. $|K|=3$. Here $K \notin \mathcal{F}, d\left(\mathcal{C}_{2}\right)=d\left(\mathcal{C}_{1}^{d}\right) \leq 3$, and Lemma 3.4 implies $p_{1} \leq 1$. The surplus of the top set is 5 , so $p_{1}=1$ and $p_{1}\left(\mathcal{C}^{d}\right) \geq 2$. But, this means $p_{3} \geq 1$ and $s(\mathcal{C}) \geq 7 \geq d(\mathcal{C})$.

4. $4 \leq|K| \leq 6$. We may assume that $|K|=4$. Here $d(\mathcal{C})=d\left(\mathcal{C}_{0}\right)+d\left(\mathcal{C}_{1}\right)$. As the surplus of the top set is 6 , and $p_{1} \geq 2$ implies $K \cup\{a, b, c\} \in \mathcal{F}$, we may assume $K \in \mathcal{F}$. We now have $s(\mathcal{C}) \geq 10$, and $d\left(\mathcal{C}_{1}\right)>5$. Therefore, $p_{1} \geq 3$. When $p_{1}=4, \mathcal{C} \subseteq \mathcal{F}$, and $d(\mathcal{C})<s(\mathcal{C})$. When $p_{1}=3$ and $K \cup\{d\} \notin \mathcal{F}$, then $s\left(\mathcal{C}_{2}\right)=3$ and $s(\mathcal{C}) \geq 13>d(\mathcal{C})$. Finally, if $p_{1}=3$ and $K \cup\{d\} \in \mathcal{F}$, then $s\left(\mathcal{C}_{3}\right) \geq 7$ and $s(\mathcal{C}) \geq 13>d(\mathcal{C})$. 
5. $|K|=0$ and $|K|=7$. The total deficit of the two hypercubes is at most $d(\emptyset)+$ $d(X \backslash\{a, b, c, d\})=s(X)+s(\{a, b, c, d\})$.

Lemma 3.8. Let $|X|=11$ and $\mathcal{F}$ contain a three-element set and a four-element set which do not intersect. Then $\mathcal{F}$ is Frankl's.

Proof. Let $\{a, b, c\},\{d, e, f, g\} \in \mathcal{F}$. The weight function will be $w(x)=2$ for $x \in$ $\{a, b, c, d, e, f, g\}$ and $w(x)=1$ for all other $x \in X$. The target weight is 9 . Let $\mathcal{C}$ be an $\{a, b, c, d, e, f, g\}$-hypercube with bottom set $K$. We will prove that the only hypercube with $d(\mathcal{C})-s(\mathcal{C})>0$ is the bottom one, and that this difference will be covered by the extra surplus in the top hypercube. The cases are:

1. $|K|=0$. Here we will prove $d(\mathcal{C}) \leq s(\mathcal{C})+8$. By Lemmas 3.7 and 3.4, any level 4 set in $\mathcal{F}$ different from $\{d, e, f, g\}$ has a two-element intersection with $\{d, e, f, g\}$. However, by Lemma 3.4 there can be at most one such set of the type $\{x, y, d, e\}$ in $\mathcal{F}$, and it implies that $\{a, b, c, d, e\} \in \mathcal{F}$. Therefore, $p_{4} \leq p_{5}+1$. So $d(\mathcal{C})=$ $d\left(\mathcal{C}_{0}\right)+d\left(\mathcal{C}_{3}\right)+d\left(\mathcal{C}_{4}\right)=9+3+p_{4} \leq p_{5}+13=s\left(\mathcal{C}_{5}\right)+s\left(\mathcal{C}_{7}\right)+8 \leq s(\mathcal{C})+8$.

2. $|K|=1$. According to Lemmas 3.7 and 3.4, every level 3 set in $\mathcal{F}$ has either a two-element intersection with $\{a, b, c\}$ (we denote their number by $q_{3}$ ), or a twoelement intersection with $\{d, e, f, g\}$ (we denote their number by $r_{3}$ ). According to Lemma 3.4, at most one of the sets $K \cup\{a, b, x\}, x \in\{d, e, f, g\}$, could be in $\mathcal{F}$ (and it implies $K \cup\{a, b, d, e, f, g\} \in \mathcal{F}$ ), hence $q_{3} \leq p_{6}$. Similarly, at most one of the sets $K \cup\{d, e, x\}, x \in\{a, b, c\}$, could be in $\mathcal{F}$, which implies $r_{3} \leq p_{5}$. Therefore, $d(\mathcal{C})=d\left(\mathcal{C}_{3}\right)=2 p_{3}=2 q_{3}+2 r_{3} \leq 4 p_{6}+2 p_{5} \leq s(\mathcal{C})$.

3. $|K|=2$. We have $d(\mathcal{C})=d\left(\mathcal{C}_{2}\right)+d\left(\mathcal{C}_{3}\right)=3 p_{2}+p_{3}$. We define $q_{3}$ and $r_{3}$ similarly as in the case $|K|=1$, while $s_{3}$ is the number of level 3 sets in $\mathcal{F}$ which have no intersection with $\{a, b, c\}$. Similarly as in the previous case, $\{d, e, f, g\},\{a, b, c\} \in \mathcal{F}$ imply that $q_{3}+4 s_{3} \leq 4 p_{6}$ and $r_{3} \leq 3 p_{5}$. Therefore, $s(\mathcal{C})-s\left(\mathcal{C}_{4}\right)-d\left(\mathcal{C}_{3}\right) \geq 7+5 p_{6}+$ $3 p_{5}-q_{3}-r_{3}-s_{3}-1 \geq 6$. Lemma 3.4 implies that the union of any two level 2 sets is a level 4 set, so $d\left(\mathcal{C}_{2}\right)>6$ implies $p_{2}=3 \leq p_{4}$, and $d\left(\mathcal{C}_{2}\right)-s\left(\mathcal{C}_{4}\right) \leq 6$.

4. $|K|=3$. Here $d(\mathcal{C})=d\left(\mathcal{C}_{1}\right)+d\left(\mathcal{C}_{2}\right)=4 p_{1}+2 p_{2}$. Let $q_{2}, r_{2}, s_{2}$ denote the number of level 2 sets in $\mathcal{F}$ whose intersection with $\{a, b, c\}$ has 0,1 and 2 elements, respectively. We have $r_{2} \leq 12$, while $\{d, e, f, g\},\{a, b, c\} \in \mathcal{F}$ imply $4 q_{2}+r_{2} \leq 4 p_{5}$, $r_{2} \leq 3 p_{4}$ and $s_{2} \leq p_{6}$. Hence, $d\left(\mathcal{C}_{2}\right)=2 q_{2}+2 r_{2}+2 s_{2} \leq 2 p_{4}+4 p_{5}+6 p_{6}+\frac{1}{3} r_{2} \leq$ $s\left(\mathcal{C}_{4}\right)+s\left(\mathcal{C}_{5}\right)+s\left(\mathcal{C}_{6}\right)+4$. On the other hand, Lemma 3.4 implies $p_{1} \leq 1$, so $d\left(\mathcal{C}_{1}\right) \leq s\left(\mathcal{C}_{7}\right)-4$

5. $|K|=4$. Note that the sets $K, K \cup\{a, b, c\}, K \cup\{d, e, f, g\}$, and $X$ have total surplus by at least 8 greater that the deficit (when all are in $\mathcal{F}$ ). We will prove that the deficit of the remaining sets in $\mathcal{C}$ is not greater than the surplus. Denote by $p_{3}^{\prime}$ the number of level 3 sets in $\mathcal{F}$ different from $K \cup\{a, b, c\}$ and by $p_{4}^{\prime}$ the number of level 4 sets in $\mathcal{F}$ different from $K \cup\{d, e, f, g\}$. Define $q_{2}, r_{2}$ and $s_{2}$ as above, let $q_{1}$ be the number of level 1 sets in $\mathcal{F}$ which have empty intersection 
with $\{a, b, c\}$ and $r_{1}=p_{1}-q_{1}$. Let $\mathcal{C}_{x}$ be the $\{d, e, f, g\}$-hypercube with bottom set $K \cup\{x\}, x \in\{a, b, c\}$. Clearly, $p_{1}\left(\mathcal{C}_{x}\right) \leq p_{2}\left(\mathcal{C}_{x}\right)+1$, and if $\mathcal{C}_{x} \cap \mathcal{F} \neq \emptyset$, then $d\left(\mathcal{C}_{x}\right)=3 p_{0}\left(\mathcal{C}_{x}\right)+p_{1}\left(\mathcal{C}_{x}\right) \leq 3+p_{2}\left(\mathcal{C}_{x}\right)+1 \leq s\left(\mathcal{C}_{x}\right)$. Note that the top set of $\mathcal{C}_{x}$ is a level 5 set of $\mathcal{C}$ which contains $\{d, e, f, g\}$, while in the proof of $q_{2} \leq p_{5}$ in the previous case we used the level 5 sets which contain $\{a, b, c\}$, so we have $3 r_{1}+r_{2}+q_{2} \leq p_{3}^{\prime}+5 p_{5}$. We also have that $q_{1} \leq p_{4}^{\prime}$ and $s_{2} \leq p_{6}$. Therefore, $d\left(\mathcal{C}_{1}\right)+d\left(\mathcal{C}_{2}\right) \leq p_{3}^{\prime}+3 p_{4}^{\prime}+5 p_{5}+7 p_{6}$.

Lemma 3.9. Let $|X|=11$ and $\mathcal{F}$ contain a three-element set. Then $\mathcal{F}$ is Frankl's.

Proof. Let $\{a, b, c\} \in \mathcal{F}$. The weight function will be $w(a)=w(b)=w(c)=4$ and $w(x)=1$ for all other $x \in X$. The target weight is 10 . Let $\mathcal{C}$ be an $\{a, b, c\}$-hypercube with bottom set $K$. There are several possible cases:

1. $|K|=1$ or $|K|=2$. In these hypercubes $d(\mathcal{C})=0$.

2. $|K|=3$. By Lemmas 3.6 and $3.4, d(\mathcal{C})=3 p_{1} \leq 3<s\left(\mathcal{C}_{3}\right)$.

3. $|K|=4$. By Lemma $3.8, d(\mathcal{C})=2 p_{1} \leq 6=s\left(\mathcal{C}_{3}\right)$.

4. $5 \leq|K| \leq 7$. We will only examine the case $|K|=5$. Obviously, $p_{1} \leq p_{2}+1$, so $d(\mathcal{C})=5 p_{0}+p_{1} \leq 3 p_{2}+7 p_{3}=s(\mathcal{C})$.

5. $|K|=0$ or $|K|=8$. The first hypercube has $d(\mathcal{C})=s(\mathcal{C})+8$ and the second one $d(\mathcal{C})=2 p_{0} \leq 10 p_{3}-8 \leq s(\mathcal{C})-8$.

Lemma 3.10. Let $|X|=11$ and $\mathcal{F}$ contain a five-element set and one of its four-element subsets. Then $\mathcal{F}$ is Frankl's.

Proof. Let $\{a, b, c, d\},\{a, b, c, d, e\} \in \mathcal{F}$. The weight function will be $w(a)=w(b)=$ $w(c)=w(d)=w(e)=2$ and $w(x)=1$ for all other $x \in X$. The target weight is 8 . Let $\mathcal{C}$ be an $\{a, b, c, d, e\}$-hypercube with bottom set $K$. We consider the following cases:

1. $|K|=1$. According to Lemma $3.4, d(\mathcal{C})=d\left(\mathcal{C}_{3}\right)=p_{3} \leq 2<3=s\left(\mathcal{C}_{5}\right)$.

2. $|K|=2$. According to Lemma $3.4, d(\mathcal{C})=d\left(\mathcal{C}_{2}\right)=2 p_{2} \leq 4=s\left(\mathcal{C}_{5}\right)$.

3. $|K|=3$. According to Lemma $3.4, d(\mathcal{C})=d\left(\mathcal{C}_{1}\right)+d\left(\mathcal{C}_{2}\right)=3 p_{1}+p_{2} \leq p_{2}+3$. Since $s\left(\mathcal{C}_{5}\right)=5$, we have $d\left(\mathcal{C}_{2}\right)>2$ and $p_{2}>2$. Now, $p_{2}=p_{1}\left(\mathcal{C}^{e}\right)$, or $K \cup\{a, b, c, d\} \in \mathcal{F}$, either way $p_{4} \geq 1$. From inequality (2) we get $3 p_{2} \leq 2 p_{3}+10$, which gives $p_{2} \leq p_{3}+3$. Combining the above results, we get $d(\mathcal{C}) \leq p_{2}+3 \leq p_{3}+3 p_{4}+5 p_{5}=s(\mathcal{C})$.

4. $|K|=4$ or $|K|=5$. We will only consider the case $|K|=4$. In this hypercube $p_{0} \leq p_{4}$ and $p_{1} \leq p_{3}+2$, so $d(\mathcal{C})=4 p_{0}+2 p_{1} \leq 4 p_{4}+2 p_{3}+4 \leq s(\mathcal{C})-2$.

5. $|K|=0$ or $|K|=6$. The first hypercube has $d(\mathcal{C})=s(\mathcal{C})+8$ and the second one $d(\mathcal{C})=2 p_{0} \leq 10 p_{5}-8 \leq s(\mathcal{C})-8$. 
Lemma 3.11. Let $|X|=11$ and $\mathcal{F}$ contain a four-element set. Then $\mathcal{F}$ is Frankl's.

Proof. Let $\{a, b, c, d\} \in \mathcal{F}$. The weight function will be $w(a)=w(b)=w(c)=w(d)=2.5$ and $w(x)=1$ for all other $x \in X$. The target weight is $t(w)=8.5$. Let $\mathcal{C}$ be an $\{a, b, c, d\}$-hypercube with bottom set $K$. We will consider the following cases:

1. $|K|=1$. Here $d(\mathcal{C})=0$.

2. $|K|=2$. According to Lemma $3.4, d(\mathcal{C})=d\left(\mathcal{C}_{2}\right)=1.5 p_{2} \leq 3<s\left(\mathcal{C}_{4}\right)$.

3. $|K|=3$. Lemma 3.4 implies $d(\mathcal{C})-s\left(\mathcal{C}_{4}\right)=3 p_{1}+0.5 p_{2}-4.5 \leq 0.5 p_{2}-1.5$, so $p_{2}>3$. Hence, $p_{3} \geq 3$ and $s\left(\mathcal{C}_{3}\right) \geq 6>d(\mathcal{C})-s\left(\mathcal{C}_{4}\right)$.

4. $|K|=4$. Lemma 3.10 implies that $p_{1}=0$ or $p_{0}=0$. If $p_{1}=0$ then $d(\mathcal{C})=d\left(\mathcal{C}_{0}\right) \leq$ $s\left(\mathcal{C}_{4}\right)$. If $p_{0}=0$, since $s\left(\mathcal{C}_{4}\right)=5.5$ and $d\left(\mathcal{C}_{1}\right)=2 p_{1}$, it must be $p_{1} \geq 3$. This implies $p_{2} \geq 3, p_{3} \geq 1$ and $s(\mathcal{C}) \geq 10>6 \geq d(\mathcal{C})$.

5. $|K|=5$ or $|K|=6$. We will only consider the case $|K|=5$. There $s\left(\mathcal{C}_{4}\right)=6.5$, which implies $d(\mathcal{C})>6.5$ which holds only if $K \in \mathcal{F}$ and $p_{1}=4$. But then $\mathcal{C} \subseteq \mathcal{F}$ and $d(\mathcal{C}) \leq s(\mathcal{C})$.

6. $|K|=0$ and $|K|=7$. The first hypercube has $d(\mathcal{C})=s(\mathcal{C})+7$ and the second one $d(\mathcal{C})=1.5 p_{0} \leq 8.5 p_{5}-7 \leq s(\mathcal{C})-7$.

Theorem 3.1. If $|X|=11$ then $\mathcal{F}$ is Frankl's.

Proof. If there are no five element sets in $\mathcal{F}$ then for every $A \in \mathcal{F}$ it holds $|A|>\frac{|X|}{2}$ and $\mathcal{F}$ is clearly Frankl's. Let $\{a, b, c, d, e\} \in \mathcal{F}$. The weight function we choose is $w(a)=w(b)=w(c)=w(d)=w(e)=2$ and $w(x)=1$ for all other $x \in X$. The target weight is 8 . Let $\mathcal{C}$ be an $\{a, b, c, d, e\}$-hypercube with bottom set $K$. There are several cases we need to consider.

1. $|K|=1$ or $|K|=2$. Here $d(\mathcal{C})=0$.

2. $|K|=3$. Here $d(\mathcal{C})-s\left(\mathcal{C}_{5}\right)=d\left(\mathcal{C}_{2}\right)-5=p_{2}-5$, so $p_{2} \geq 6$. Hence, $p_{3} \geq 2$ and $p_{4} \geq 1$, which gives $s(\mathcal{C}) \geq 10 \geq p_{2}=d(\mathcal{C})$.

3. $|K|=4$. Here $d(\mathcal{C})-s\left(\mathcal{C}_{5}\right)=d\left(\mathcal{C}_{1}\right)-6=2 p_{1}-6$, so $p_{1} \geq 4$. Hence, $p_{3} \geq 4$ and $p_{4} \geq 1$, which gives $s(\mathcal{C}) \geq 18>d(\mathcal{C})$.

4. $|K|=5$. Here $d(\mathcal{C})-s\left(\mathcal{C}_{5}\right)=d\left(\mathcal{C}_{0}\right)+d\left(\mathcal{C}_{1}\right)-7=3+p_{1}-7$, so $p_{1}=5$. Hence, $\mathcal{C} \subseteq \mathcal{F}$, which gives $s(\mathcal{C}) \geq d(\mathcal{C})$.

5. $|K|=0$ and $|K|=7$. The first hypercube has $d(\mathcal{C})=s(\mathcal{C})+6$ and the second one $d(\mathcal{C})=2 p_{0} \leq 8 p_{5}-6 \leq s(\mathcal{C})-6$.

\section{Acknowledgements}

The authors wish to thank Professor Theresa P. Vaughan for many useful comments. 


\section{References}

[1] W. Gao and H. Yu, Note on the Union-Closed Sets Conjecture. Ars Combin. 49 (1998), pp. 280-288.

[2] K. Henry and M. S. Roddy, A Notion of Reducibility for Union-Closed Families of Sets. (in preparation)

[3] R. T. Johnson and T. P. Vaughan, On Union-Closed Families, I. J. Combin. Theory Ser. A 84 (1998), no. 2, pp. 242-249.

[4] G. Lo Faro, A Note on the Union-Closed Sets Conjecture. J. Austral. Math. Soc. Ser A 57 (1994), no. 2, pp. 230-236.

[5] G. Lo Faro, Union-Closed Sets Conjecture: Improved Bounds. J. Combin. Math. Combin. Comp. 16 (1994), pp. 97-102.

[6] P. Marković An Attempt at Frankl's Conjecture. Proceedings of the Novi Sad Algebraic Conference 2005 (NSAC'05), a special issue of Publ. Math. Inst. (Beograd) (N. S.) 81(95) (2007), pp. 29-43.

[7] R. Morris FC-families and Improved Bound for Frankl's Conjecture. European J. Combin. 27 (2006), no. 2, pp. 269-282.

[8] B. Poonen, Union-Closed Families. J. Combin. Theory Ser. A 59 (1992), no. 2, pp. 253-268.

[9] I. Rival (ed.), Graphs and Order. NATO Advanced Science Institute Series C: Mathematical and Physical Sciences, 147 D. Reidel Publishing Co., Dordrecht-Boston, Mass. (1985), p. 525.

[10] R. P. Stanley, Enumerative Combinatorics, Vol. I. The Wadsworth and Brooks/Cole Mathematics Series The Wadsworth and Brooks/Cole Advanced Books and Software, Monterey, Calif. (1986).

[11] T. P. Vaughan, Families Implying the Frankl Conjecture. European J. Combin. 23 (2002), no. 7, pp. 851-860.

[12] T. P. Vaughan, A Note on the Union-Closed Sets Conjecture. J. Combin. Math. Combin. Comput. 45 (2003), pp. 95-108.

[13] T. P. Vaughan, Three-sets in a Union-Closed Family. J. Combin. Math. Combin. Comput. 49 (2004), pp. 73-84. 